\title{
Kinesin-1-dependent transport of the $\beta$ PIX/GIT complex in neuronal cells
}

\author{
Eun-Young Shin ${ }^{1}$, Chan-Soo Lee ${ }^{2}$, Han-Byeol Kim ${ }^{1}$, Jin-Hee Park ${ }^{1}$, Kwangseok Oh ${ }^{1}$, Gun-Wu Lee ${ }^{1}$, Eun-Yul Cho ${ }^{1}$, \\ Hyong Kyu Kim ${ }^{3}$ \& Eung-Gook Kim, ${ }^{1, *}$ \\ Departments of ${ }^{1}$ Biochemistry and ${ }^{3}$ Microbiology, College of Medicine, and Medical Research Center, Chungbuk National University, \\ Cheongju 28644, ${ }^{2}$ Food Standard Division Scientific Office, Ministry of Food and Drug Safety (KFDA), Cheongju 28159, Korea
}

\begin{abstract}
Proper targeting of the $\beta$ PAK-interacting exchange factor ( $\beta \mathrm{PIX} / \mathrm{G}$ protein-coupled receptor kinase-interacting target protein (GIT) complex into distinct cellular compartments is essential for its diverse functions including neurite extension and synaptogenesis. However, the mechanism for translocation of this complex is still unknown. In the present study, we reported that the conventional kinesin, called kinesin-1, can transport the $\beta P I X / G I T$ complex. Additionally, $\beta$ PIX bind to KIF5A, a neuronal isoform of kinesin-1 heavy chain, but not KIF1 and KIF3. Mapping analysis revealed that the tail of KIF5s and LZ domain of $\beta$ PIX were the respective binding domains. Silencing KIF5A or the expression of a variety of mutant forms of KIF5A inhibited $\beta$ PIX targeting the neurite tips in PC12 cells. Furthermore, truncated mutants of $\beta \mathrm{PIX}$ without $\mathrm{LZ}$ domain did not interact with KIF5A, and were unable to target the neurite tips in PC12 cells. These results defined kinesin-1 as a motor protein of $\beta P I X$, and may provide new insights into $\beta$ PIX/GIT complex-dependent neuronal pathophysiology. [BMB Reports 2021; 54(7): 380-385]
\end{abstract}

\section{INTRODUCTION}

Beta PAK (p21-activated kinase)-interacting exchange factor ( $\beta \mathrm{PIX})$ is a guanine nucleotide exchange factor (GEF) for Rac1/Cdc42 GTPases (1) that regulates the dynamics of lamellipodial and filopodial actin structures in response to extracellular cues. Two distinct genes encode the mammalian PIX family members namely $\alpha \mathrm{PIX}$ and $\beta \mathrm{PIX}(2,3)$. $\alpha \mathrm{PIX}$ and $\beta \mathrm{PIX}$ have similar domain structures, with the exception of an extra calponin

*Corresponding author. Tel: +82-43-261-2848; Fax: +82-43-272-1603; E-mail: egkim@chungbuk.ac.kr

https://doi.org/10.5483/BMBRep.2021.54.7.061

Received 10 May 2021, Revised 18 May 2021, Accepted 31 May 2021

Keywords: $\beta$ PAK-interacting exchange factor ( $\beta$ PIX), G proteincoupled receptor kinase-interacting target protein (GIT), Kinesin-1, Neuron, Transport homology $(\mathrm{CH})$ domain at the $\mathrm{N}$-terminus of $\alpha \mathrm{PIX}$ (4). $\beta \mathrm{PIX}$ interacts stably and transiently with $\mathrm{G}$ protein-coupled receptor kinase-interacting protein (GIT) and Rac1/Cdc42 effector p21activated kinase (PAK), respectively, thus generating a highmolecular weight complex designated here as the $\beta \mathrm{PIX}$ complex $(5,6)$.

The $\beta$ PIX complex plays a key role in a wide range of biological processes, including neurite extension (7-9) and cell migration $(9,10)$, which requires the correct subcellular localization of the complex. The neuronal growth cone, found at the tip of axonal or dendritic projection, is a motile structure whose migration promotes neurite extension during the development of the nervous system and regeneration. At the growth cone, mostly near the actin-rich peripheral zone, the $\beta$ PIX complex localizes and promotes growth cone motility $(8,11)$. The $\beta P I X$ complex can also be found at synapses, in both presynaptic and postsynaptic areas, causing synaptogenesis including dendritic spine morphogenesis (12-15). The $\beta$ PIX complex promotes neurite extension and synaptogenesis through dynamic cytoskeletal rearrangement. In contrast to these well-known functions of the $\beta$ PIX complex, the mechanism for its subcellular translocation is still unknown.

Motor protein kinesin-1 (also known as conventional kinesin-1) mediates plus end-directed, microtubule-dependent transport. Several types of cargo for kinesin-1 has been identified, including protein complexes, organelles, and mRNA (16). Kinesin-1 is a tetramer composed of two identical kinesin heavy chains (KHCs) and two identical kinesin light chains (KLCs) (17). The mammalian genome contains three $\mathrm{KHC}$ genes namely KIF5A, $\mathrm{KIF5B}$, and KIF5C. Among these $\mathrm{KHC}$ isoforms, KIF5A and KIF5C are neuron-specific, whereas KIF5B is expressed ubiquitously (18). Two major cargo-interaction sites have been recognized: (i) the $\mathrm{C}$-terminal heptad-repeat region in the tail domain of $\mathrm{KHC}(19,20)$ and (ii) the C-terminal tetratricopeptide repeat region in KLC (21). The former is responsible for direct binding to kinectin, RanBP2, SNARE proteins, GRIP1 and $\beta$-dystrobrevin (22).

In view of the critical role of the $\beta$ PIX complex in diverse biological processes, we aim to understand its targeting mechanism by identifying a motor protein capable of transporting this complex. The conventional kinesin-1 was identified as an interacting 
partner of $\beta$ PIX. By using biochemical and imaging analyses, we demonstrated that $\beta$ PIX binds directly to KIF5 which is a heavy chain subunit of kinesin-1. $\beta$ PIX interacted with all three isoforms of KIF5 through their tail domains. Thus, kinesin-1 may function as a universal transporter of the $\beta$ PIX complex in diverse types of cells.

\section{RESULTS}

\section{BPIX binds directly to KIF5A}

To identify a molecular motor that transports the $\beta$ PIX complex, we performed affinity purification using an anti-BPIX antibody and mass spectrometry of distinctively silver-stained bands. A band around $120 \mathrm{kD}$ was identified as KIF5A, a neuronal isoform of kinesin heavy chain (KHC) (Fig. 1A, left), which was confirmed by immunoblotting (Fig. 1A, right). To confirm the association between BPIX and KIF5A, a series of co-immunoprecipitation assays were conducted. From the results, it was observed that KIF5A and KLC were co-precipitated with $\beta P I X$ and GIT1 in the lysates from rat brains (Fig. 1B), cultured hippocampal neuron cells (Supplementary Fig. 1A) and PC12 cells (Supplementary Fig. 1B). However, no immunoprecipitation was noted between $\beta \mathrm{PIX}$ and KIF1A and KIF3A, indicating specificity of binding. PAK2 was detected upon precipitation

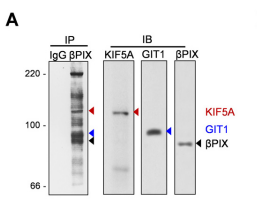

D

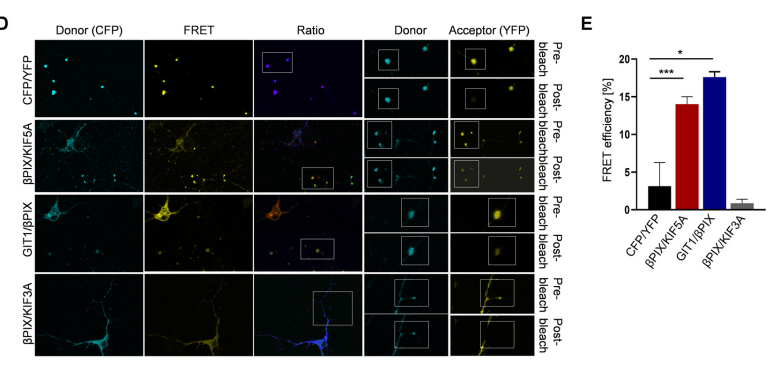

Fig. 1. Association of $\beta P I X$ with KIF5A in the rat brain. (A) Mass spectrometry. Anti- $\beta P I X$ immunoprecipitates were silver stained (left). Marked silver-stained bands (arrowheads) were subjected to mass spectrometry followed by immunoblotting (right, three columns). (B) Immunoblotting for the indicated proteins following immunoprecipitation with anti-3PIX, -KIF5A, or -KLC antibody. (C) GST pulldown assay. Histagged proteins (input and bound), Glutathione-Sepharose immobilized GST-ßPIX or GST protein (bead) were immunoblotted by antiHis, or GST antibody. (D) Representative FRET images from primary cultured rat hippocampal neurons co-transfected with plasmids encoding the indicated pairs of proteins. Acceptor (YFP)-bleached areas were indicated by white boxes. Negative control, CFP/YFP and $\beta P I X-$ CFP/KIF3A-YFP; positive control, GIT1-CFP/BPIX-YFP. (E) Quantification of FRET efficiencies (mean \pm SEM.). $\mathrm{N}=20$ cells/each condition. Student's $t$-test. ${ }^{*} \mathrm{P}=0.0124, * * * \mathrm{P}<0.0001$ when using anti- $\beta P I X$ antibody. However, when using anti-KIF5A or anti-KLC antibodies, detection of PAK2 was not possible (Fig. 1B, Supplementary Fig. 1A, B). Further analysis revealed an interaction of $\alpha \mathrm{PIX}$ complex with KIF5A. Fusion proteins of green fluorescent protein (GFP)- $\alpha$ PIX or $\beta P I X$ co-precipitated with KIF5A, but no co-precipitation was noted with GFP alone (Supplementary Fig. 1C). Taken together, these results indicated that both $\alpha \mathrm{PIX}$ and $\beta \mathrm{PIX}$ complexes specifically interacted with KIF5A.

Since $\beta$ PIX makes a tight complex with GIT1, RNAi technology was used to determine whether it was $\beta$ PIX or GIT1 which was responsible for interacting with KIF5A. When PC12 cells were treated with specific siRNAs for $\beta$ PIX or GIT1, immunoblotting detected a marked downregulation of their expression (Supplementary Fig. 2). However, no GIT1 was detected in the KIF5A immunoprecipitates from $\beta$ PIX-depleted cells (Supplementary Fig. 2A, middle), showing that there is no direct association between GIT1 and KIF5A. Conversely, silencing GIT1 did not impact the association of $\beta$ PIX with KIF5A (Supplementary Fig. $2 \mathrm{~B}$, top), confirming the importance of $\beta \mathrm{PIX}$ in binding to KIF5A.

To confirm the interaction between KIF5A and $\beta$ PIX at molecular level, fluorescence resonance energy transfer (FRET) analysis was conducted in hippocampal cells using the acceptor bleaching method. In FRET measurement, the donor and acceptor were CFP- and YFP-tagged, respectively. If a donor and acceptor protein were spatially arranged in close proximity, the intensity of the acceptor (YFP) fluorescence would increase over time following the photobleaching of the acceptor (YFP). Two pairs, CFP/YFP and $\beta P I X / K I F 3 A$, were used as negative controls as they do not exhibit biochemical interactions (Fig. 1B). No significant FRET was detected from the pair of CFP/YFP $(3.13 \pm 3.13 \%)$ or $\beta$ PIX-CFP/KIF3A-YFP $(0.87 \pm 0.53 \%)$ (Fig. $1 \mathrm{D}, 1^{\text {st }}$ and $4^{\text {th }}$ row; quantified in E). GIT1 directly bound to the GIT binding domain (GBD) of $\beta$ PIX via its Spa2 homology domain (SHD) and coiled-coiled region. Thus, the pair of GIT1-CFP/BPIX-YFP can function as a positive control. This pair showed a higher FRET efficiency (17.6 \pm $0.72 \%$ ) (Fig. 1D, $3^{\text {rd }}$ row; quantified in E). The FRET efficiency for $\beta$ PIX-CFP/KIF5A was $14.05 \pm 1.09 \%$ as comparable to the positive pair of GIT1/BPIX (Fig. 1D, $2^{\text {nd }}$ row; quantified in E). Collectively, these results indicated that $\beta$ PIX indeed interacted with KIF5A in the rat brain and in cultured cells.

\section{Analysis of the binding domains that mediated interaction between KIF5A and $\beta$ PIX}

A mapping analysis was conducted to determine which part of KIF5A/BPIX was responsible for binding. The motor, stalk or tail domains of KIF5A as illustrated in Fig. 2A were expressed in a His-tagged form and incubated with GST- $\beta$ PIX or GST alone (control). GST-BPIX pulled down the tail region of KIF5A (Fig. 2B, top, lane 6) while GST control showed no specific binding (lane 3). We further determined whether the tail domains from KIF5B and KIF5C interacted with $\beta$ PIX because 
A
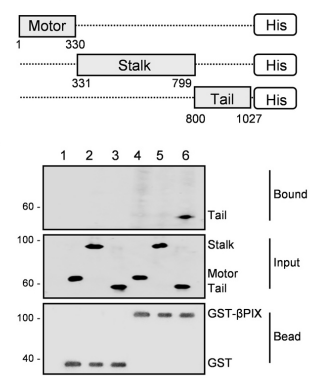

E

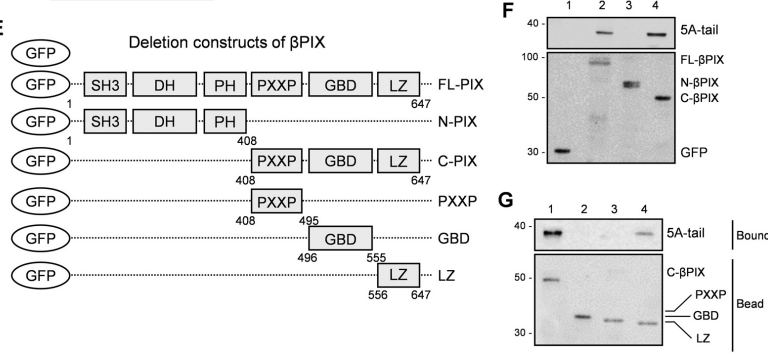

Fig. 2. Direct interaction between the $I Z$ domain of $\beta P I X$ and the tail domain of KIF5. (A) Schematic diagram of KIF5A constructs. (B, D) GST pulldown. Bound proteins were detected by immunoblotting with anti-His antibody (top). (C, E) Schematic diagram of the constructs for KIF5 tail (C) and BPIX (E). Each domain was expressed as His-tagged $(\mathrm{C})$ or GFP-fused $(\mathrm{E})$ protein. (F, G) Immunoprecipitation and immunoblotting. PC12 cells were transfected with plasmid encoding GFP-tagged $\beta$ PIX construct (bottom) followed by immunoprecipitation with anti-GFP antibody. Bound KIF5A tail was detected by immunoblotting with anti-His antibody (top).

of their high homology. Each tail of the three KIF5 isoforms was expressed as a His-tagged protein (Fig. 2C) and incubated with GST- $\beta$ PIX. Results showed that all three tail domains bound to $\beta$ PIX (Fig. 2D, top, lanes 4-6). Conversely, to identify the binding domain in $\beta P I X$, various truncated forms of $\beta$ PIX in GFP-fused proteins were produced (Fig. 2E) and transfected into PC12 cells followed by immunoprecipitation with an anti-GFP antibody. The tail of KIF5A was bound to both full-length $\beta$ PIX (FL- $\beta P I X)$ and C-terminal half of $\beta P I X(C-\beta P I X)$ but not to the $\mathrm{N}$-terminal half of $\beta \mathrm{PIX}(\mathrm{N}-\beta \mathrm{PIX})$ (Fig. $2 \mathrm{~F})$. This result prompted to explore the subdomain of C- $\beta$ PIX, thus, C- $\beta$ PIX was fragmented into three parts, PXXP, GBD and LZ domains (Fig. 2E, bottom 3 rows). Incubations of these domains with His-tagged KIF5A revealed LZ domain as the binding partner (Fig. 2G). Taken together, these results indicated that the tail domain of KIF5 and LZ domain of $\beta P I X$ mediated interaction between KIF5 and $\beta$ PIX.

\section{KIF5A-dependent targeting of $\beta$ PIX to the neurite tip and neuronal synapses}

To understand the functional significance of interaction between KIF5A and BPIX, we examined the effect of dominant negative mutants of KIF5A on targeting $\beta$ PIX to the targeted sites. For
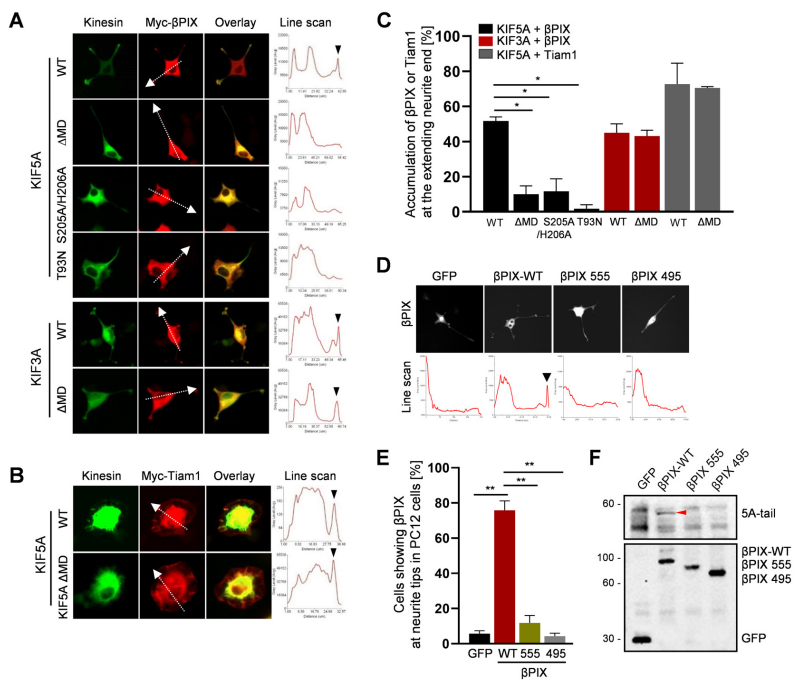

Fig. 3. KIF5A-dependent $\beta P I X$ targeting to the neurite tip (A, B). Representative images for the localization of $\beta \mathrm{PIX}$ (A) and Tiam1 (B) in differentiated PC12 cells. Kinesin constructs were GFP-tagged, and $\beta$ PIX and Tiam1 were Myc-tagged. Two days after co-transfected cells were stained with anti-GFP and anti-Myc antibodies. Arrowheads at the line scan indicate neurite tips. Note specific KIF5A-dependent localization of $\beta$ PIX at the neurite tip. (C) Quantification of the localization of $\beta$ PIX and Tiam1 in (A, B). Accumulation of $\beta$ PIX, and Tiam-1 at the neurite tip was quantified by line scan analysis using MetaMorph software. Student's $t$-test. $* P<0.05, N=30$. (D) Representative images for the localization of GFP-tagged BPIX constructs in PC12 cells. (E) Quantification of the localization of $\beta$ PIX constructs at the neurite tip in (D). Student's t-test. $* * P<0.01, N=20$. (F) Association of $\beta$ PIX constructs with KIF5A tail. PC12 cells were transfected with pEGFP- $\beta$ PIX constructs for 2 days and were lysed and immunoprecipitated with anti-GFP antibody. Immunoprecipitates were incubated with purified His-tagged KIF5A tail followed by immunoblotting with anti-His antibody. Note that only BPIX-WT interacted with KIF5A tail (red arrowhead).

this purpose, we produced KIF5A mutants without a motor domain (KIF5A $\triangle \mathrm{MD}$ ), microtubule-binding capacity (S205A H206A) or ATPase activity (T93N) that function in a dominant negative manner. Endogenous $\beta \mathrm{PIX}$ and KIF5A were abundantly expressed in the cytoplasm and co-localized at the neurite tips in differentiated PC12 cells (Supplementary Fig. 3A, top). Exogenous YFP-tagged $\beta P I X$ and GFP-tagged KIF5A showed a similar behavior (Supplementary Fig. 3A, bottom). In cells expressing those KIF5A mutants, no significant accumulation of $\beta \mathrm{PIX}$ at the neurite ends was detected, as assessed by line scan (Fig. 3A, 2-4 rows; quantified in C). Their expression also caused some morphological changes in PC12 cells. For instance, retracted neurites with slender tapering ends were observed instead of finger-shaped growth cone-like structures. Unlike KIF5A mutants, expression of a motor less KIF3A did not affect the $\beta$ PIX targeting the neurite tip. Localization of Tiam1, another Rac1 GEF, was also unaffected by a motor less KIF5A (Fig. 3B; quantified in C). These results 
suggested that KIF5A may serve as a specific motor to translocate $\beta P I X$ to the neurite tip.

The LZ domain of $\beta \mathrm{PIX}$ was responsible for binding to KIF5A (Fig. 2G). Thus, it could be difficult to properly target the neurite ends if $\beta P I X$ is constructed without LZ domain. To test this suggestion, we employed two truncated mutants of $\beta P I X$ without the LZ domain, namely $\beta$ PIX 555 (aa 1-555) and $\beta P I X$ 495 (aa 1-495) (Fig. 3D). As shown in Fig. 3D, the two mutants demonstrated a significant impairment in their migration to the neurite ends (i.e., detected in less than $11 \%$ of cells) (Fig. 3E). In contrast, more than $70 \%$ of cells expressing full-length $\beta$ PIX (WT) marked a clear peak at the targeted location (Fig. 3E). Consistent with this result, co-immunoprecipitation revealed an interaction of KIF5A with only full-length $\beta \mathrm{PIX}$ amongst the $\beta$ PIX constructs (Fig. 3F). Since $\beta$ PIX can also target neuronal synapses and can subsequently lead to synaptogenesis, its targeting ability was therefore further examined herein. Endogenous $\beta$ PIX and KIF5A co-localized in the cell body and small puncta along the dendritic neurites in cultured hippocampal neurons can be seen in Supplementary Fig. 3B, top. Exogenous $\beta$ PIX and KIF5A also showed a similar behavior, suggesting their synaptic co-localization (Supplementary Fig. 3B, bottom). To examine the targeting of GFP-fused full-length $\beta P I X(W T)$ and $\beta P I X 555$ to synapses, cells were stained for a postsynaptic marker PSD-95. The white merged dots along the dendrites represent co-localization of WT $\beta$ PIX and PSD-95 (Supplementary Fig. 4A, middle row; quantified in B). In contrast, $\beta$ PIX 555 and GFP control showed a significant decrease of approximately $50 \%$ in co-localization which could be attributed to their simple diffusion to the postsynaptic compartment. These results provided strong evidence for KIF5A as a specific transporter of $\beta$ PIX.

\section{DISCUSSION}

Precise spatiotemporal targeting of the BPIX complex to the specific subcellular locations is essential for its cellular function. This study presented a unified mechanism underlying the transport of the $\beta$ PIX complex to neurite tips and synapses in neuronal cells. We identified kinesin-1 as a binding partner of the $\beta$ PIX complex; all KHCs bound the LZ domain of $\beta$ PIX via their tail domain. Notably, both the tail and LZ domains shared a similar coiled-coil structure that facilitated the formation of protein dimers. Each binding domain thus appeared to play a key role in the formation of a large multi-complex. These conserved binding sites may also help kinesin-1 function as a transporter of the $\beta$ PIX complex.

Molecular motors such as kinesin and dynein are essential in establishing and maintaining neuronal polarity by transporting axon- or dendrite-specific cargos in a tightly regulated manner (23). Upon suppression of kinesin-1 expression by antisense oligonucleotides, hippocampal cells displayed shorter neurites accompanied by defect in transport of GAP-43 and synapsin I to the neurite tips (24). Considering its catalytic function as a
Rac1/Cdc42 GEF, KIF5-dependent targeting of the $\beta$ PIX complex to growth cone-like structures at the neurite tips was consistent with its role in neurite outgrowth and axonal neuritogenesis. Abnormal dendrites with long, thin dendritic spines are typically observed in patients with mental retardation (MR) (25). Rho GTPases play an important role in actin-based cytoskeletal changes for morphogenesis of dendritic spines. Amongst thirteen genes whose mutations caused nonspecific $X$-linked MR, three genes encoding $\alpha$ PIX, PAK3 and oligophrenin 1 were identified to directly regulate Rho GTPase signaling (26). $\beta P I X$ in the dendritic spines was reported to interact with Shank, a scaffold protein in the PSD (27), and liprin- $\alpha$ through GIT1 (28). Although $\alpha \mathrm{PIX}$ is also located in dendritic spines, its interacting partner is still unknown. Our current findings supported kinesin-1-dependent transport of both $\alpha \mathrm{PIX}$ and $\beta$ PIX. Considering their localization in the dendritic spines and similar role in actin dynamics, it seems intriguing why $\beta$ PIX does not compensate for the functional loss of $\alpha$ PIX in mentally retarded patients. $\beta$ PIX-deficient mice died during early embryonic development, but $\alpha$ PIX-deficient mice were alive and appeared normal after birth, suggesting its unknown function that cannot be compensated by $\beta$ PIX. This warrants future study to elucidate their distinct functions, in particular in the dendritic spines.

The present study has several limitations. Firstly, although $\alpha$ PIX and $\beta$ PIX bind KIF5A (Supplementary Fig. 1C) and their subcellular targeting would be kinesin-1 dependent, the present study focused on $\beta P I X$. It remains to be determined whether $\alpha P I X$ can show a similar targeting behavior like $\beta$ PIX. Secondly, it is uncertain how the PIX complex's kinesin-1-dependent mobility is controlled, for example, how to start or stop its movement. PAK2 was identified as a component of BPIX immunoprecipitates (Supplementary Fig. 1A), suggesting involvement of PAK2-mediated phosphorylation in the regulatory event. However, this kinase was not detected in KIF5A and KLC immunoprecipitates (Supplementary Fig. 1A), contradicting the former idea.

In conclusion, the present study defined kinesin-1 as a universal transporter of the $\beta \mathrm{PIX}$ complex based on the diverse binding and functional analysis. $\beta$ PIX complex is known to regulate a number of key cellular activities in neuronal cells. Understanding its transport mode in association with kinesin-1 may provide novel insight into the pathophysiological function of the $\beta$ PIX complex in neurons.

\section{MATERIALS AND METHODS}

\section{Cell culture}

PC12 cells were grown in Dulbecco's modified Eagle's medium (DMEM) supplemented with 10\% fetal bovine serum (FBS) and $100 \mathrm{U} / \mathrm{ml}$ penicillin/streptomycin (Invitrogen) and were kept at $37^{\circ} \mathrm{C}$ in a humidified $5 \% \mathrm{CO}_{2}$ incubator. Hippocampus from the brain of newborn (P1) Sprague-Dawley (SD) rat were dissected and incubated with papain in dissociation medium 
(pH 7.4) (82 $\mathrm{mM} \mathrm{Na}_{2} \mathrm{SO}_{4}, 30 \mathrm{mM} \mathrm{K} \mathrm{SO}_{4}, 5.8 \mathrm{mM} \mathrm{MgCl}$, $0.252 \mathrm{mM} \mathrm{CaCl}_{2}, 1 \mathrm{mM}$ HEPES, $20 \mathrm{mM}$ glucose, $0.5 \%$ phenol red) containing L-cystein for $20 \mathrm{~min}$ at $37^{\circ} \mathrm{C}$ and dissociated by pipetting in DMEM containing $10 \%$ FBS.

\section{Mass spectrometry}

Rat brain lysate was immunoprecipitated with anti- $\beta$ PIX antibody immobilized onto CNBr-activated sepharose 4B. Immunoprecipitates were resuspended in SDS-PAGE sample buffer and were separated by SDS-PAGE. The gel was silver stained by using a kit according to the manufacturer's protocol (Bio-Rad, CA, USA). Specific bands excised from silver stained gel were subjected to mass spectrometry by a company (Genomine, Kyungbuk, Korea).

\section{FRET microscopy}

Cultured PC12 cells were co-transfected with a pair of plasmids for FRET analysis using LF2000, incubated for 36-48 $\mathrm{h}$ and fixed in phosphate-buffered saline (PBS) containing $3.7 \%$ paraformaldehyde for $10 \mathrm{~min}$ at RT. After washing with PBS, cells were mounted onto a glass slide. FRET measurement was performed on the Leica TCS SP2 confocal microscope according to the FRET acceptor photobleaching protocol (Leica, Wetzlar, Germany). The HCX PL APO $63 \times$ objective was used and excitation was provided by $20 \mathrm{~mW}$ multimode argon in laser lines. CFP was detected at $454 \mathrm{~nm}$ and YFP was detected and photobleached at $514 \mathrm{~nm}$. Laser intensity was set to $20 \%$ for YFP detection but $100 \%$ for bleaching. The gain of the photomultiplier detectors was adjusted to obtain the optimal dynamic range. The CFP fluorescence was measured before and after YFP bleaching and FRET efficiency was calculated according to the equation; FRET efficiency [\%] = $\left(\mathrm{CFP}_{\text {after }}-\mathrm{CFP}_{\text {before }}\right) \times 100 / \mathrm{CFP}_{\text {after }}$.

\section{In vitro binding assay}

pGEX4T-1-BPIX and pET-FL-KIF5 constructs were expressed in E. coli $\mathrm{DH} 5 \alpha$ or BL21. Purified GST-FL- $\beta$ PIX were immobilized onto glutathione-Sepharose 4 beads. GST- $\beta$ PIX-immobilized beads were incubated with His-tagged FL-KIF5A, motor (aa 1-330), stalk (aa 331-799), or tail (aa 800-1027) in binding buffer (20 mM HEPES, pH 7.4, $0.15 \mathrm{M} \mathrm{NaCl}, 1 \mathrm{mM}$ DTT, $0.2 \%$ Triton X-100, $5 \mu \mathrm{M} \mathrm{MgSO}_{4}$, and protease inhibitor) for 1 $h$, and then subjected to immunoblotting with antibodies to His and GST. In some experiments, GST- $\beta$ PIX-immobilized beads were incubated with lysates from PC12 cells transfected with various $\beta \mathrm{PIX}$ constructs. Proteins bound to beads were subjected to SDS-PAGE and immunoblotted with the corresponding antibodies.

\section{Immunoprecipitation and immunoblotting}

Cells were lysed on ice with ice-cold lysis buffer $(50 \mathrm{mM}$ HEPES, pH 7.5, $150 \mathrm{mM} \mathrm{NaCl}, 1 \mathrm{mM}$ EDTA, $10 \%$ glycerol, $200 \mu \mathrm{M}$ sodium orthovanadate, $10 \mathrm{mM}$ Na-pyruvate, $50 \mathrm{mM}$ glycerophosphate, $1 \%$ triton X-100). Lysates were immunoprecipitated with a primary antibody for $2 \mathrm{~h}$ followed by incu- bation with protein G Sepharose for $5 \mathrm{~h}$. Immunoprecipitates were separated by SDS-PAGE, and transferred to PVDF membrane (Millipore, MA, USA) for $2 \mathrm{~h}$. Membranes were blocked with $4 \%$ skim milk, incubated with a primary antibody for $1 \mathrm{~h}$, and were blotted with secondary antibodies (PIERCE, IL, USA). Immunoblots were developed using enhanced chemiluminescence solution.

\section{Statistical analysis}

All experimental data were expressed as means \pm SEM. Statistical significance was assessed using an unpaired $t$-test (t-test) using GraphPad Prizm software (version 10) for Windows. $\mathrm{P}<0.05$ was considered statistically significant.

\section{ACKNOWLEDGMENTS}

This work was partially supported by the National Research Foundation of Korea (NRF) grant funded by the Korea government (MSIP) (2020R1A5A2017476 and 2020R1A2C1011976), and Bio \& Medical Technology Development Program (2017M3A9D8063627).

\section{CONFLICTS OF INTEREST}

The authors have no conflicting interests.

\section{REFERENCES}

1. Manser E, Loo TH, Koh CG et al (1998) PAK kinases are directly coupled to the PIX family of nucleotide exchange factors. Mol Cell 1, 183-192

2. Oh WK, Yoo JC, Jo D, Song YH, Kim MG and Park D (1997) Cloning of a SH3 domain-containing proline-rich protein, p85SPR, and its localization in focal adhesion. Biochem Biophys Res Commun 235, 794-798

3. Bagrodia S, Taylor SJ, Jordon KA, Van Aelst L and Cerione RA (1998) A novel regulator of p21-activated kinases. J Biol Chem 273, 23633-23636

4. Kim S, Lee SH and Park D (2001) Leucine zipper-mediated homodimerization of the p21-activated kinase-interacting factor, beta Pix. Implication for a role in cytoskeletal reorganization. J Biol Chem 276, 10581-10584

5. Premont RT, Perry SJ, Schmalzigaug R, Roseman JT, Xing $Y$ and Claing A (2004) The GIT/PIX complex: an oligomeric assembly of GIT family ARF GTPase-activating proteins and PIX family Rac1/Cdc42 guanine nucleotide exchange factors. Cell Signal 16, 1001-1011

6. Schlenker O and Rittinger K (2009) Structures of dimeric GIT1 and trimeric beta-PIX and implications for GIT-PIX complex assembly. J Mol Biol 386, 280-289

7. Shin EY, Shin KS, Lee CS et al (2002) Phosphorylation of p85 beta PIX, a Rac/Cdc42-specific guanine nucleotide exchange factor, via the Ras/ERK/PAK2 pathway is required for basic fibroblast growth factor-induced neurite outgrowth. J Biol Chem 277, 44417-44430

8. Shin EY, Lee CS, Yun CY et al (2014) Non-muscle myosin II regulates neuronal actin dynamics by interacting with 
guanine nucleotide exchange factors. PLoS One 9, e95212

9. Za L, Albertinazzi C, Paris S, Gagliani M, Tacchetti $C$ and de Curtis I (2006) betaPIX controls cell motility and neurite extension by regulating the distribution of GIT1. J Cell Sci 119, 2654-2666

10. Lee CS, Choi CK, Shin EY, Schwartz MA and Kim EG (2010) Myosin II directly binds and inhibits Dbl family guanine nucleotide exchange factors: a possible link to Rho family GTPases. J Cell Biol 190, 663-674

11. Mo J, Lee D, Hong $S$ et al (2012) Preso regulation of dendritic outgrowth through $\mathrm{PI}(4,5) \mathrm{P} 2$-dependent PDZ interaction with betaPix. Eur J Neurosci 36, 1960-1970

12. Llano O, Smirnov S, Soni S et al (2015) KCC2 regulates actin dynamics in dendritic spines via interaction with beta-PIX. J Cell Biol 209, 671-686

13. Kim S, Ko J, Shin $\mathrm{H}$ et al (2003) The GIT family of proteins forms multimers and associates with the presynaptic cytomatrix protein Piccolo. J Biol Chem 278, 6291-6300

14. Saneyoshi T, Wayman G, Fortin D et al (2008) Activitydependent synaptogenesis: regulation by a CaM-kinase kinase/CaM-kinase I/betaPIX signaling complex. Neuron 57, 94-107

15. Shin MS, Song SH, Shin JE, Lee SH, Huh SO and Park D (2019) Src-mediated phosphorylation of betaPix-b regulates dendritic spine morphogenesis. J Cell Sci 132, 1-17

16. Hirokawa N, Noda Y, Tanaka Y and Niwa S (2009) Kinesin superfamily motor proteins and intracellular transport. Nat Rev Mol Cell Biol 10, 682-696

17. Bloom GS, Wagner MC, Pfister KK and Brady ST (1988) Native structure and physical properties of bovine brain kinesin and identification of the ATP-binding subunit polypeptide. Biochemistry 27, 3409-3416

18. Kanai $Y$, Okada Y, Tanaka Y, Harada A, Terada S and Hirokawa N (2000) KIF5C, a novel neuronal kinesin enriched in motor neurons. J Neurosci 20, 6374-6384

19. Kirchner J, Seiler S, Fuchs S and Schliwa M (1999) Functional anatomy of the kinesin molecule in vivo. EMBO J 18, 4404-4413

20. Seiler S, Kirchner J, Horn C, Kallipolitou A, Woehlke G and Schliwa M (2000) Cargo binding and regulatory sites in the tail of fungal conventional kinesin. Nat Cell Biol 2, 333-338

21. Gindhart JG Jr and Goldstein LS (1996) Tetratrico peptide repeats are present in the kinesin light chain. Trends Biochem Sci 21, 52-53

22. Gindhart JG (2006) Towards an understanding of kinesin-1 dependent transport pathways through the study of proteinprotein interactions. Brief Funct Genomic Proteomic 5, 74-86

23. Nakata T and Hirokawa N (2007) Neuronal polarity and the kinesin superfamily proteins. Sci STKE 2007, pe6

24. Ferreira A, Niclas J, Vale RD, Banker G and Kosik KS (1992) Suppression of kinesin expression in cultured hippocampal neurons using antisense oligonucleotides. J Cell Biol 117, 595-606

25. Purpura DP (1974) Dendritic spine "dysgenesis" and mental retardation. Science 186, 1126-1128

26. Ramakers GJ (2002) Rho proteins, mental retardation and the cellular basis of cognition. Trends Neurosci 25, 191-199

27. Park E, Na M, Choi J et al (2003) The Shank family of postsynaptic density proteins interacts with and promotes synaptic accumulation of the beta PIX guanine nucleotide exchange factor for Rac1 and Cdc42. J Biol Chem 278, 19220-19229

28. Ko J, Kim S, Valtschanoff JG et al (2003) Interaction between liprin-alpha and GIT1 is required for AMPA receptor targeting. J Neurosci 23, 1667-1677 